\title{
OPTIMAL RESTORATION OF POWER SUPPLY IN LARGE DISTRIBUTION SYSTEMS IN DEVELOPING COUNTRIES
}

\author{
V. Susheela Devi \\ D.P. Sen Gupta, Senior Member, IEEE \\ Department of Electrical Engineering \\ Indian Institute of Science \\ Bangalore, India
}

\begin{abstract}
A computer aided optimal method has been developed for the restoration of electric supply to areas isolated from the network following a fault in a distribution system. A search technique is used where the search is guided by appropriate heuristics. The optimum solution entails finding the strategy which involves the operation of minimum number of switchgear for rerouting the supply within the constraint of specified loading. This is an essential requirement in countries like India where the circuit breakers are almost always manually operated and a number of transformers and feeders operate close to their rated capacity. It pays therefore to adopt different strategies at peak load and off peak conditions since the number of breaker operations is so critical. The heuristic search that is developed is applied to a large distribution system and provides very good results.
\end{abstract}

\section{I: INTRODUCTION}

It is imperative that power supply be restored rapidly following a fault. This paper provides a methodology for restoring electric power in a very large distribution system in a developing country.

This paper was presented at the 1994 IEEE PES Transmission and Distribution Conference and Exposition held in Chicago, Illinois, April 10-15, 1994.

\author{
G. Anandalingam, Senior Member, IEEE \\ Dept. of Systems Engineering \\ University of Pennsylvania \\ Philadelphia, PA 19104
}

The restoration of electric supply to isolated areas of large-scale urban distribution systems is a difficult task. When a fault occurs on a feeder, the faulted section has to be first identified and isolated. The isolated sections will have to be fed from alternative feeders until the faulty cable is repaired, a task that could take some time. The restoration of supply to isolated sections involves the opening and closing of switchgear so as to feed the isolated sections from alternative routes. It is necessary that in the process of restoring power, there is no overloading of buses, transformers, and line sections.

The present practice of restoring supply in developing countries is for the operating personnel to make the decision on the spot, relying on past experience. It is difficult for the operating personnel to keep in mind the entire system, especially if it is large, and changing and growing all the time. The memory and computational speed of the computer can be very usefully exploited to offer feasible solutions. The final decision can rest with the operator. The program will aid the personnel in obtaining the optimal solution quickly.

In this paper we provide a heuristic search based methodology for optimally restoring electric power supply. This methodology is the basis of computer software that has been developed and applied to a large sub-grid in Bombay(India) city.

A number of different approaches have been suggested for solving the problem of restoring electric supply after the onset of a fault that interrupts it. The literature on power supply restoration is surveyed in the next section. The methods proposed are integer programming which is difficult to implement for large distribution systems, and expert systems, which do not as yet have the scope to deal with complexities in a developing country. This paper provides better results than those in the liter- 
ature, particularly for the case of developing countries. The methodology is similar to that in the paper by Morelato and Monticelli[7] in that a tree search methodology is adopted. However there are significant differences in our paper. First, we provide very different heuristics for solving the problem and find that breadth-first, rather than depth-first is more suitable for our purposes. Secondly, we prune the search tree, and thus save considerably on computation time. Thirdly, we provide realistic results in the context of a developing country.

Most importantly, to our knowledge, the network to which we have applied the methodology is easily the largest in the literature.

In a developing country, switchgear operation is mostly manual, not controlled by a computer. In India, for instance, the-switchgear operators are dispatched by bicycle to the location of the switches that need to be turned on or off. Thus, while load balancing etc. are important, most of the cost is related to the number of switches that need to be operated. Thus in our model, the objective is to minimize the number of switchgear operations.

Next, since service restoration is manual, the time taken to restore operations is not as small as it would be in situations where computer control is used. Hence, it is imperative that service be restored as quickly as possible. Trying to find a solution to restore power supply so that peak load for the next 24 hour period is met will take a significant amount of time. Also, it will lead to a large number of manual switching operations. The strategy of restoring supply soon, in order to meet the load expected in the next few hours, and then, after the system stabilizes, resorting to peak-load satisfaction may be a better strategy. Thus, in this paper, we experiment with a number of strategies for restoring power that may or may not satisfy peak-load requirements.

The paper is organized as follows: In section 2 we provide a brief literature survey. Section 3 presents the heuristic search techniques that we have derived in this paper. We give details of the different algorithms that are required to implement our heuristic search in section 4 . Section 5 discusses the different load considerations that we have analyzed in the paper. In section 6 , we provide numerical results on applying our methodology to a large distribution network. We end our paper with some concluding remarks in section 7 .

\section{LITERATURE SURVEY}

The interest on the subject of restoration of supply in distribution systems following a fault is relatively recent. Aoki et al. $[1,2]$ have employed integer programming to minimize the load magnitude in a feeder. Whenever there is a fault, the de-energized loads have to be transferred to neighboring feeders. The decision on which switches are to be operated and which feeders to transfer the load to, are decided by using integer programming. A computer program was run on a system consisting of 5 substations, 11 transformers, 86 feeders and 577 switches of which 198 are normally-open.

Switching operations are frequently carried out on the basis of human expert judgement in most parts of the world. Sakaguchi et al. [9] have used INTERLISP for the expert system. A set of 16 rules are used for supply restoration for a very small system of 9 buses and 14 lines. Liu et al. [4] have an expert system in PROLOG with 180 rules. Here the outage area is divided into groups, each group to be fed from a different feeder. Each group load is transferred to a neighboring feeder when supply interruption occurs. The transferring of load follows a set of rules based on reserve margin of capacity of the lines. This expert system was used for a small system of $12.5 \mathrm{kV}$ comprising 3 substations, 6 primary feeders, 43 sections and 6 open tie switches. The authors claim that the restoration procedure takes a few seconds to obtain a solution. S.J. Lee et al. [6] have tried to improve upon the expert system as reported in [4]. The number of rules is 382 with some enhanced capabilities.

Another technique that has been used to derive solutions to the problem of restoring power has been heuristic search. The problem space consists of $(0,1)$ variables that determine whether a switch should be off or on. Morelato et al.[7], use a binary search tree and depth-first search. The solution is chosen to minimize the Load Balancing Index. The method was implemented only for a small system. Castro et al.[3] have tried the restoration procedure by searching through the switch table which contains information about the status of the switches in the system. Although the time to find a solution is less than $10 \mathrm{msec}$, the program has not been used for large systems. 
Other search procedures include Ross et al. [8] and Dialynas et al.[5]. The largest system for which these methods were used consisted of 115 nodes, 10 feeders and a single substation. The time taken to obtain a solution was between 3 to 37 seconds.

\section{HEURISTIC SEARCH TECHNIQUE}

\section{A. Overview}

The restoration procedure consists of providing a sequence of connection and disconnection of sections for restoring supply to the areas that lose electric power when a fault occurs. Before restoring supply it is necessary to ascertain that there is no overloading anywhere in the system. The loading on all sections, feeders, buses and transformers should be within their capacity.

We also want the system to be as balanced as possible. This can be achieved by transferring the load whenever possible to a feeder with a greater margin. We wish to minimize the number of switch operations under the constraints of capacity and load balancing.

\section{B. The Methodology}

The search tree consists of switches that can be opened or shut in order to restore electric supply to the affected region. At the initiation of the search, the normally-open switches which can feed the isolated sections are first ascertained. These involve just a single operation, and in the search tree are of path length one. If any of these solutions is feasible, i.e., if none of the distribution links are overloaded, the task is over. Otherwise these form partial solutions, and other routes are found to relieve the overload. The strategy for relieving overload is to split the isolated section, and to feed it by two normally open switches. If two open switches do not exist or it is not feasible, then the overload on the host feeder is relieved by transferring the extra load to a neighboring feeder. This solution set forms nodes of path length two. If these solutions are not feasible, then the nodes can be expanded further until a feasible solution is found. When a feasible solution is found, it is necessary to trace back through the tree to find the complete sequence of operations.

The number of switchgears to be operated increases with the path length. So the deeper one goes into the tree, the larger is the number of switchgear that needs to be operated. Thus, the optimal solution will be obtained if one is at a "shallow" part of the search tree.

There are usually three broad ways in which one controls heuristic search of solution trees: depthfirst, breadth-first, and best-first. From the preceding argument it is clear that depth-first search is likely to be inefficient, i.e. the computation time to obtain minimum switch operation will be long. Since breadth-first search first examines all the nodes on one level before examining any of the nodes at the next level, this procedure should give the optimum solution faster than other search techniques.

Best-First Search: In general, using best-first search yields solutions faster than that using breadth-first alone. In best-first search, nodes chosen for expansion depends on which feeder has minimum overload.

If $L_{f}$ is the load on a feeder $f$ and $C_{f}$ is the capacity of the feeder $f$ then the excess load to be relieved will be $\left(L_{f}-C_{f}\right)$. At each step, the node $i$ with minimum overloading is chosen for expansion. This means that node $i$ is chosen so as to minimize $\left(L_{f i}-C_{f i}\right)$. It is to be noted that if

$$
\left(L_{f i}-C_{f i}\right)<0
$$

it means that there is no overloading and we have a feasible solution.

By always expanding the most likely node, this procedure makes it possible to get a solution much faster even if it lies deep down in the tree. The problem is that best-first search does not always give the optimal solution. It is possible that an unexplored path would have yielded a solution with fewer number of operations. Thus, optimality is sacrificed for the sake of increased speed. In our application to the real world system, using best-first procedure outlined above speeded up execution time. However, it was found that on some occasions, optimality was not obtained.

\section{Pruning Of The Search Tree}

Although breadth-first search is the obvious strategy for minimizing operation of switchgear, it may be time consuming, especially for very large systems. One method of reducing the search is to dis- 
card paths which are unlikely to offer a solution. We provide a heuristic for pruning the search tree.

In most cases, loads $\left(P_{L}\right)$ in the post-fault region have to be transferred to another feeder $X$. If feeder $X$ is overloaded due to the transfer, a section $T$ from $X$ has to be transferred to a third feeder $Y$ to prevent overloading of $X$. Suppose $P_{T}$ is the sum of the loads of section $T$, we use the pruning rule that

$$
P_{T}<a P_{L}
$$

where $a$ is found experimentally to be of the order of 2 or 3 ; i.e., any search that leads to an excessive accumulation of the loads to be transferred should be anticipated and abandoned. With these values of $a$, it was found that the time taken for the search was reduced and, for the distribution system we analyzed the results were found to be identical with one exception, to those for breadth-first search without pruning. For details of the justification of using $a=2$ or 3 , see [10].

\section{Load Flow and Capacity Considerations}

In order to ascertain the feasibility of any solutions, it is necessary to check if the loading of the transformers, feeders and line sections are within the capacity. This can be ascertained by doing a load flow calculation. Whenever a load is transferred to a feeder, a load flow calculation is carried out only for the feeder which is taking the excess load. A simple DC load flow calculation is used. This was considered adequate since only the loading on the system is being checked. In cases where voltage drop or the system losses need to be calculated, a DC load flow is obviously inadequate. By not using exact AC calculations some amount of accuracy is sacrificed.

\section{E. Example System}

The methodology explained in the preceding sections is best understood by an example. Figure 1 shows a small system consisting of 8 feeders. For a fault in section $6288-7149$ of feeder 7149 , Figure 2 shows the resulting search tree. The area beyond the node 6288 in feeder 7149 is thus without supply. The order in which the tree develops (figure 2) depends on the type of search used. The numbers in parenthesis show the development of the tree using breadth-first search. In best-first search, all nodes at level 1 are first found. Then the node where minimum load needs to be transferred is expanded to level 2. These nodes at level 2 and the remaining nodes at level 1 are then checked, and the node where minimum load needs to be transferred is again chosen for expansion. Whenever a solution is found to be feasible, the complete solution can be found by backtracking up the tree. For example, in figure 2 if the node at (21) is the right solution, the complete solution will be

$\begin{array}{ll}\text { CONNECT } & 6972-1817 \\ \text { DISCONNECT } & 7791-8108 \\ \text { CONNECT } & 6148-8323 \\ \text { DISCONNECT } & 7193-5120 \\ \text { CONNECT } & 7196-27196\end{array}$

\section{IMPLEMENTATION OF THE ALGORITHM}

\section{A. Overview}

The heuristic search algorithm presented in this paper has been implemented on an IBM PC using the TURBO PASCAL language. The program has been tested out on a very large system consisting of over 2500 nodes and 120 feeders.

The system considered is the actual metropolitan distribution system of an electric utility in the city of Bombay in India. As a result of the large size of the system, there were problems of inadequate memory space.

Some space saving measures had to be adopted to accommodate voluminous data. For details of data structures, see the thesis by Susheela Devi[10].

\section{B. Algorithmic Details}

Figure 3 captures the interactions of the algorithms. For details, see [10].

Algorithm 'CONNECT' assigns the distribution nodes to the feeders to which they belong.

Algorithm 'ISOLATE' is used to check to see if the disconnected node has the same connectivity number as any of the starting node of the feeders. Algorithm ' $A L T$ ' is used to locate the normallyopen sections that may feed this group of nodes.

When a load is transferred to a feeder, the feeder may get overloaded. Algorithm ' $C U T$ ' is used to de- 
cide where to cut the feeder and transfer the overload beyond the cut section.

The algorithm ' $L O A D$ ' computes the aggregate load on each feeder. The algorithm ' $L O A D F L O W$ ' carries out the DC load flow for a feeder after which it checks for any overloading in any of the sections.

Finally, algorithm ' $D I S P$ ' is used to display a part of the map. The feeders which have to be drawn are specified.

\section{Multiple Faults}

The program is capable of handling multiple faults. Multiple faults result in there being multiple isolated areas which have to be fed from alternative routes. These isolated areas are handled one at a time in sequence, until all parts of the network are restored. For details, see Susheela Devi[10]

\section{EFFECT OF LOAD ON RESTORATION}

Loads can also be classified into various categories like commercial load, residential load, heavy industrial load, light industrial load etc. The typical daily load curves for the various categories are required if supply restoration was to give priority to specific users. We have not done priority supply restoration in this paper. It is evident that with growing demand it will become increasingly difficult to find solutions to restore supply if peak load is used. Using peak load gives a solution which is valid at all times of the day. However, large number of manual operations may be required to restore supply which may be difficult to carry out within a short time, especially during the night. The strategy of restoring supply immediately, based on off-peak load and reverting to the one based on peak load (if the solution is different) may ease pressure on the personnel and the affected customers. In our analysis we examine different levels of demand to be restored.

\section{RESULTS}

\section{A. Data Used}

The system used is fairly large. Figure 1 gives a small portion of the actual system consisting of 8 feeders. For details, see[10].

The entire system being about 15 times larger consisting of 120 feeders and over 2500 nodes. There are about 180 normally-open sections which can be connected for the restoration of supply. Each link has isolating switches so that any section in the system can be disconnected. There are 29 transformers $(66 / 11 \mathrm{kV})$ feeding the whole area. We should note that this is to our knowledge the largest distribution system which has been examined in the literature on power supply restoration.

\section{B. Comparison of Different Heuristics}

Faults have been chosen at random, and solutions have been obtained using different heuristics: breadth-first, breadth-first with pruning, and bestfirst. A comparison has been made of the time required to obtain a solution and the number of switchgear operation involved in the various solutions that were obtained. The number of trials required to obtain a solution gives a fairly accurate picture of the time required.

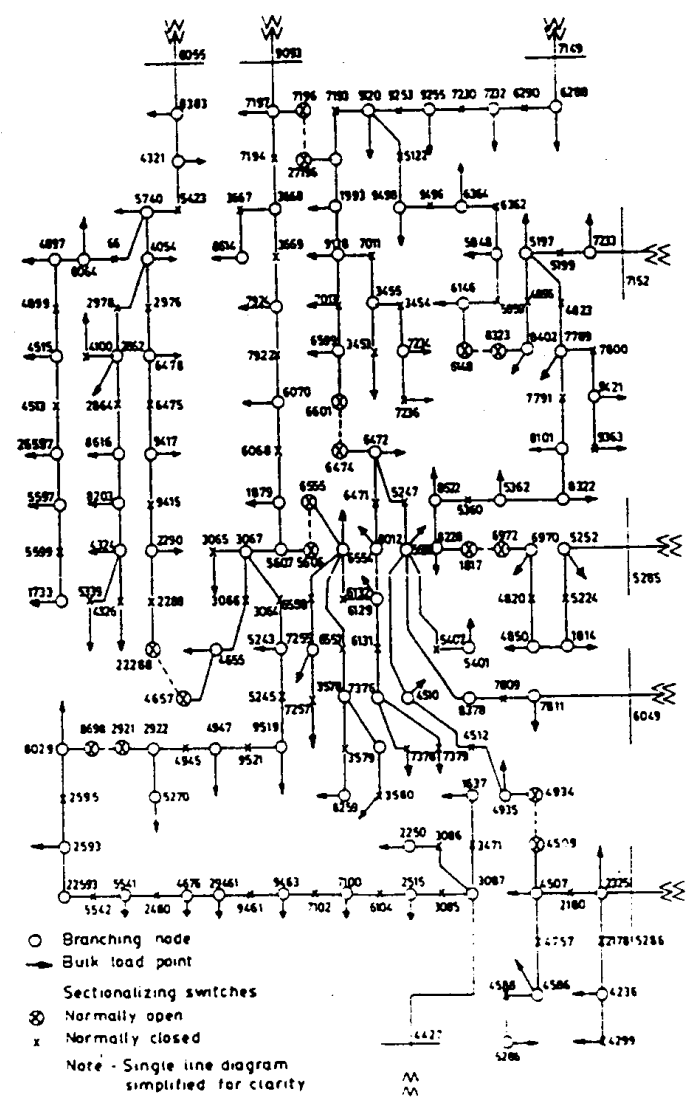

Figure 1: Example Distribution System 


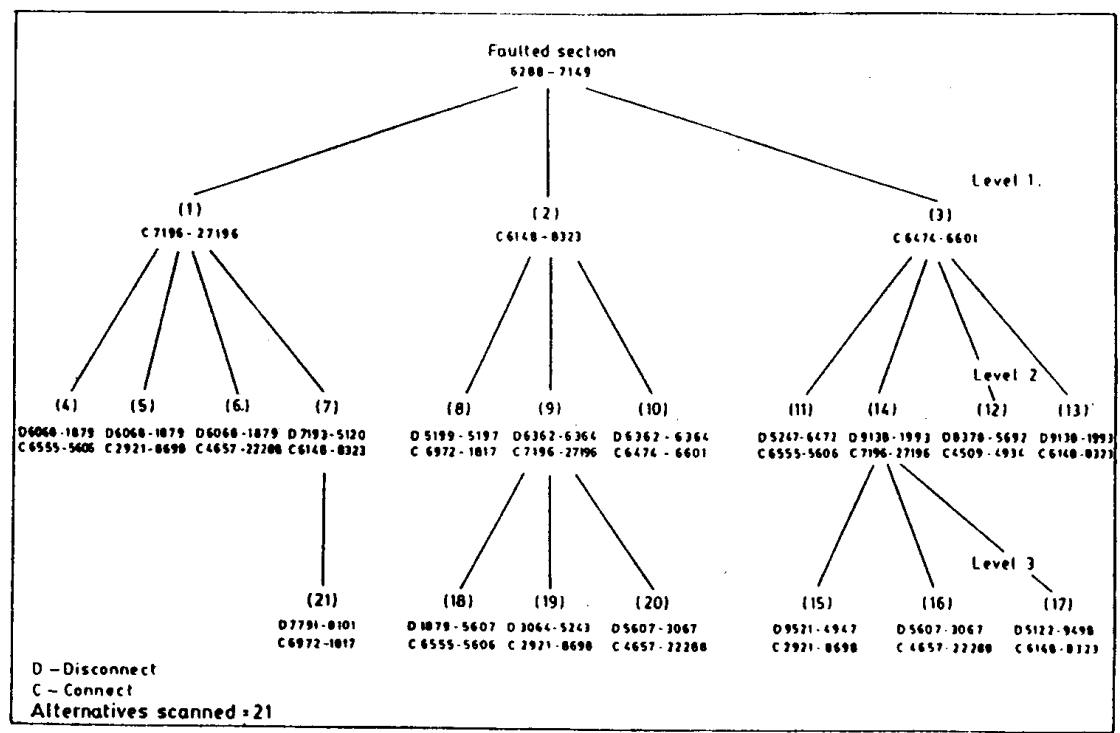

Figure 2: Search Tree for the Restoration Process in Example

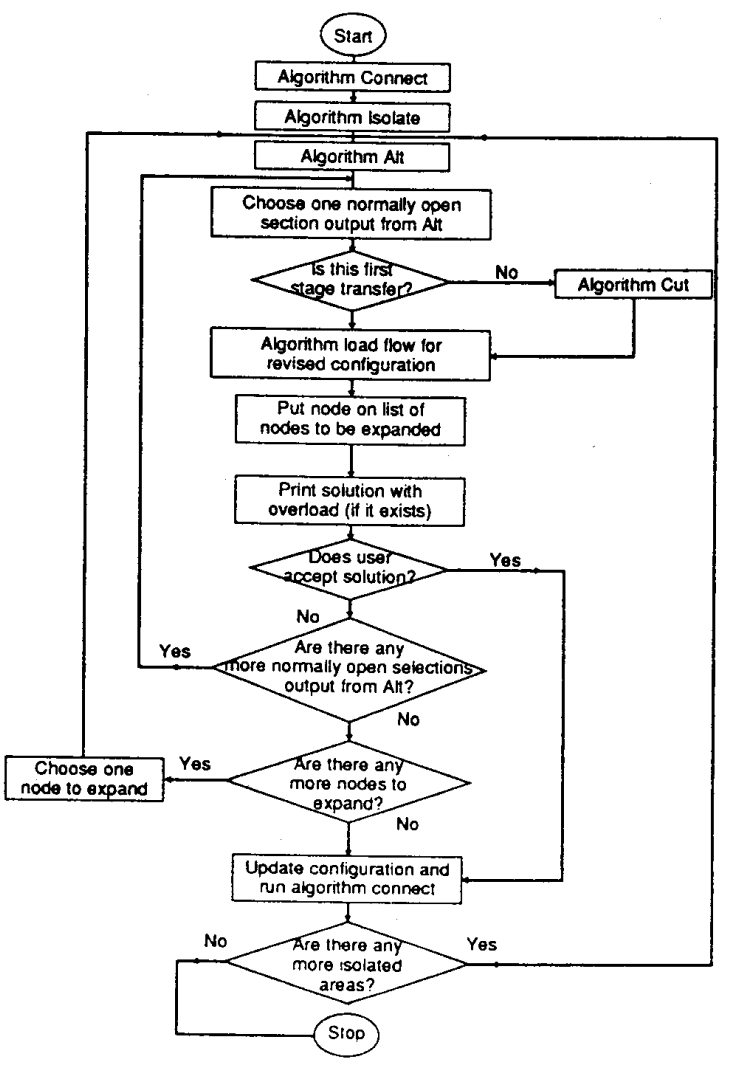

Figure 3: Flow Chart for the Restoration Process 
Table 1 gives selected results. It can be seen that best-first search gives results much faster than the breadth-first search. This method appears to be preferable especially in cases where the number of trials required for the breadth-first search is very large. It was however, seen that the best-first search may occasionally converge to a sub-optimal solution as evident in the first trial. The breadth-first search converged after 64 iterations while the best first search converged after 23 iterations and took significantly less time. The latter, however, gave a solution involving more switchgear operations (5 operations). If the search tree is pruned, the number of paths searched reduces. As mentioned earlier, the pruning rule is $P_{T} \leq a P_{L}$. Using $a=2$ means that more paths are being pruned than if $a=3$. Pruning provides a slight improvement in the number of trials needed to obtain the solution. Also the solutions were almost always optimal (i.e. coincident with breadth-first results).

From our computational experience, and knowledge of the system it seems clear that the best first search is to be preferred in situations when, (a) the fault is close to the bus, (b) the feeders are already loaded and the margin available is limited, (c) the cables are to be derated due to ageing.

For simpler cases there is hardly any reason to choose one method over the other. If the number of operations is of critical importance and the loading is relatively light for example, at night) the breadth first search may be used because it is guaranteed to obtain the optimal solution.

\section{The Effect of Loading}

We examined the implications of load growth on the number of switch operations to restore interrupted power supply by assuming an annual load growth of 10 per cent. The results obtained for some of the faults is given in Table 2 which shows that for some faults, as expected after the load grows, the solutions involve operation of large number of switchgear (over nine switchgear operations). We also examined the implications of using the maximum load in the next few hours for performing the supply restoration instead of the peak load. Depending on the area and the time of the day the loads were approximately estimated and the computations carried out. Some results are presented in Table 3. For different faults, the solutions were obtained at 6 hours, 12 hours, 18 hours and 24 hours. In almost all cases (except Sl. Nos. 6 and 9), two additional switching operations are required for restoring supply on the occurrence of faults if the maximum load in the next 18 hours was considered rather than the maximum load in the next 6 , 12 and 24 hours. For manual switching operation even the reduction of 2-3 switching operations is of considerable significance especially at night hours. The line restoration obtained during the night may in real practice be allowed to continue beyond off peak hours (with possibly marginal overloading of one or two feeders).

\section{CONCLUDING REMARKS}

We have presented a technique for the restoration of electric power supply on the occurrence of a fault on large distribution systems. The search is guided by simple heuristics, limited by specific knowledge of the area and constraints that exist and provided accurate results very rapidly. We recommend using breadth-first with pruning. If the time required for obtaining the solution is very critical, then the method can be used in advance of an actual fault. A few alternative strategies computed by this method can be easily stored in the computer for a few anticipated locations, and as soon as the exact fault location is communicated to the operator, he would be ready with a switching strategy.

Sometimes it may not be possible to feed all areas after a fault occurs. In such a case it will be necessary to resort to load shedding. In such a contingency, it should be ensured that minimum load is shed and certain loads are spared. A priority ordering of the nodes has not been considered in this paper. If the nodes are given priority ordering (hospitals and essential services having high priority for instance), then load shedding can be carried out according to the priority of the nodes.

Another approach is for the algorithm to 'learn' the suitable criteria for optimality and the appropriate control strategies by the use of some examples. In addition, the practical experience of the operating personnel can be built into the program in order to guide the search. There are many ways of improving the heuristic search procedure which is reported in this paper. This is the focus of ongoing research. 


\section{REFERENCES}

[1] Aoki, K., Kuwabara, H., Satoh, T., Kanezashi, M., "Outage state optimal load allocation by automatic sectionalizing switches operation in distribution systems," IEEE Trans on Power Delivery, Vol. PWRD-2, No. 4, pp. 1177 - 1185, October 1987.

[2] Aoki, K., Nara, K., Itoh, M., Satoh, T., Kuwabara, H., "A new algorithm for service restoration in distribution systems," IEEE Trans on Power Delivery, Vol. 4, No. 3, pp. 1832 1839, July 1989.

[3] Castro, Carlos H., Bunch, Jennings B., Topka, Terry M., "Generalized algorithms for distribution feeder deployment and sectionalizing," IEEE Trans on PAS, Vol. PAS-99, No. 2, pp. 549 - 557, March/April 1980.

[4] Chen-Ching Liu, Seung Jae Lee, S.S. Venkata, "An expert system operational aid for restoration and loss reduction of distribution systems," IEEE Trans on Power Systems, Vol. 3, No. 2, pp. 619 - 626, May 1988.

[5] Dialynas, E.N., Michos, D.G., "Interactive modelling of supply restoration procedures in distribution system operation," IEEE Trans on Power Delivery, Vol. 4, No. 3, pp 18471854 July 1989.

[6] Lee, S.J., Liu, C.C., Venkata, S.S., "An extended expert system for service restoration of distribution feeders," IFAC Symposium, Power Systems, Modelling and Control Applications, pp. 16.4.1 - 16.4.7, September 1988.

[7] Morelato, A.L., Monticelli, A., "Heuristic search approach to distribution system restoration," IEEE Trans on Power Delivery, Vol. 4, No. 4, pp. 2235 - 2241, October 1989.

[8] Ross, Dale W., Patton, James, Cohen, Arthur I., Carson, Marlyn, "New methods for evaluating distribution automation and control(DAC) systems benefit," IEEE Trans on PAS, Vol. PAS-100, No. 6, pp. 2978 - 2986, June 1981.

[9] Sakaguchi, T., Matsumoto, K., "Development of a knowledge based system for power system restoration", IEEE Trans PAS, Vol. PAS-22, No. 2, pp. 320 - 329, February 1983.

[10] Susheela Devi, "Optimal restoration of electric supply following a fault on large distribution systems," M.Sc. (Engg.) thesis, Department of Electrical Engineering,Indian Institute of Science, Bangalore, January 1992.

\section{BIOGRAPHIES}

V. Susheela Devi graduated from the Bangalore University in 1982 and completed her Masters from the Indian Institute of Science, Bangalore. At present she works at the Department of Electrical Engineering, Indian Institute of Science.

D.P. Sen Gupta graduated from IIT, Kharagpur in 1961 and did his Ph.D. from the University of Liverpool after two years' experience in AEI, Manchester. He was a Lecturer in the University of Liverpool for four years and joined the Indian Institute of Science, Bangalore in 1970. He was promoted as Professor in 1979. He has had research links with the University of Liverpool, Queen's University, Belfast and Cavendish Laboratory, Cambridge. He has authored three books and a number of technical papers. Real time control of instability in power systems, distribution networks and rural energy problems in India have been his recent areas of research.

G. Anandalingam received the B.A. degree in electrical sciences in 1975 from Cambridge; the M.S. and Ph.D. degrees in Systems Engineering from Harvard in 1977 and 1981 respectively. He is an associate professor at the Department of Systems at the University of Pennsylvania, Philadelphia, PA. He is also Director of the ExMSE (Masters Degree in Executive Engineering) program for Moore Fellows in the management of technology. He is on the Editorial Board of Computers and Operations Research and Telecommunications Systems 
Table 1: Pruning of Search Tree



Table 2: Cases Showing Difficulty in Obtaining a Solution as the Load Increases

\begin{tabular}{cccccccc}
\hline $\begin{array}{c}\text { Sl. } \\
\text { No. }\end{array}$ & $\begin{array}{l}\text { Faulted } \\
\text { Section }\end{array}$ & \multicolumn{6}{c}{ number of switchgear operated } \\
& & $\begin{array}{c}100 \% \text { of } \\
\text { peak load }\end{array}$ & $\begin{array}{c}110 \% \text { of } \\
\text { peak load }\end{array}$ & $\begin{array}{c}121 \% \text { of } \\
\text { peak load }\end{array}$ & $\begin{array}{c}133 \% \text { of } \\
\text { peak load }\end{array}$ & $\begin{array}{c}146 \% \text { of } \\
\text { peak load }\end{array}$ & $\begin{array}{c}161 \% \text { of } \\
\text { peak load }\end{array}$ \\
\hline 1. & $2022-4429$ & 1 & 1 & 3 & $>9$ & $>9$ & $>9$ \\
2. & $3436-6192$ & 3 & 3 & 3 & 5 & 5 & 9 \\
3. & $1739-7561$ & 3 & 3 & 3 & 5 & 5 & $>9$ \\
4. & $6288-7149$ & 5 & $>9$ & $>9$ & $>9$ & $>9$ & $>9$ \\
5. & $3071-5189$ & 1 & 1 & 7 & 7 & $>9$ & $>9$ \\
\hline
\end{tabular}

Table 3: Results with Time of Day Loading

\begin{tabular}{|c|c|c|c|c|c|c|c|c|c|c|}
\hline \multirow{2}{*}{$\begin{array}{l}\text { Sl. } \\
\text { No. }\end{array}$} & \multirow{2}{*}{$\begin{array}{l}\text { Faulted } \\
\text { Section }\end{array}$} & \multirow{2}{*}{$\begin{array}{l}\text { Percent of } \\
\text { peak load }\end{array}$} & \multicolumn{3}{|c|}{6 hours } & \multicolumn{2}{|l|}{12 hours } & \multicolumn{2}{|l|}{18 hours } & \multirow{2}{*}{$\begin{array}{l}24 \text { hours } \\
\text { No. of } \\
\text { Oper. }\end{array}$} \\
\hline & & & $\begin{array}{l}\text { No. of } \\
\text { trials }\end{array}$ & $\begin{array}{l}\text { No. of } \\
\text { Oper. }\end{array}$ & $\begin{array}{l}\text { No. of } \\
\text { trials }\end{array}$ & $\begin{array}{l}\text { No. of } \\
\text { Oper. }\end{array}$ & $\begin{array}{l}\text { No. of } \\
\text { trials }\end{array}$ & $\begin{array}{c}\text { No. of } \\
\text { Oper. }\end{array}$ & $\begin{array}{l}\text { No. of } \\
\text { trials }\end{array}$ & \\
\hline 1. & $3436-6192$ & 133 & 13 & 3 & 13 & 3 & 23 & 5 & 5 & 3 \\
\hline 2. & $6288-7149$ & 100 & 4 & 3 & 4 & 3 & 12 & 7 & 4 & 3 \\
\hline 3. & $2325-5286$ & 100 & 1 & 1 & 1 & 1 & 8 & 5 & 1 & 1 \\
\hline 4. & $3071-5189$ & 121 & 5 & 5 & 3 & 3 & 11 & 7 & 1 & 1 \\
\hline 5. & $1822-5437$ & 121 & 4 & 3 & 4 & 3 & 14 & 5 & 1 & 1 \\
\hline 6. & $1981-5963$ & 121 & 1 & 1 & 1 & 1 & 1 & 1 & 1 & 1 \\
\hline 7. & $1739-7561$ & 133 & 4 & 3 & 4 & 3 & 8 & 5 & 3 & 3 \\
\hline 8. & $25536-6056$ & 100 & 11 & 3 & 11 & 3 & 27 & 5 & 11 & 3 \\
\hline 9. & $7233-7152$ & 100 & 1 & 1 & 1 & 1 & 1 & 1 & 1 & 1 \\
\hline 10. & $6460-6527$ & 146 & 5 & 3 & 5 & 3 & 6 & 5 & 1 & 1 \\
\hline
\end{tabular}

